\title{
Effect of GnRH antagonists on clinical pregnancy rates in ovulation induction protocols with gonadotropins and intrauterine insemination
}

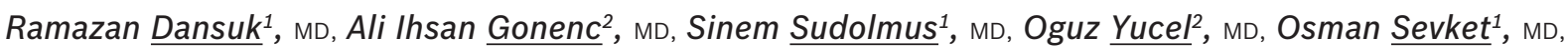
Nadiye $\underline{\text { Köroğlu }}^{1}, \mathrm{MD}$

INTRODUCTION Intrauterine insemination (IUI) after controlled ovarian hyperstimulation (COH) was applied to selected infertile patients to determine the effect of gonadotropin-releasing hormone (GnRH) antagonists in IUI cycles, in which recombinant follicle-stimulating hormone ( $\mathrm{rFSH}$ ) had been used for $\mathrm{COH}$.

METHODS This study was conducted between April 1, 2009 and June 10, 2009, and involved a total of 108 patients. These patients had primary or secondary infertility, which resulted in an indication for IUI, and they each received two cycles of ovarian stimulation treatment with clomiphene citrate. The patients were randomised into two groups - patients in group A received $\mathrm{rFSH}+\mathrm{GnRH}$ antagonist $(n=45)$, while those in group B received only $\mathrm{rFSH}(n=63)$.

RESULTS The mean age of the patients was $31.84 \pm 3.73$ years and the mean body mass index (BMI) was $24.40 \pm 1.88 \mathrm{~kg} / \mathrm{m}^{2}$. The mean age and BMI of the patients in groups A and B were not significantly different. There was no significant difference in the mean total rFSH dose administered (988.33 IU in group A and $871.83 \mathrm{IU}$ in group B). When compared to group $B$, the mean number of follicles that were $>16 \mathrm{~mm}$ on the human chorionic gonadotropin (HCG) trigger day was significantly higher in group A $(1.58$ and 1.86 , respectively; $p<0.05)$. When the two groups were compared, there were no statistically significant differences in the number of cancelled cycles due to premature luteinisation (none in group A vs. two in group B) and the rate of clinical pregnancy ( $8.9 \%$ in group A vs. $7.9 \%$ in group B).

CONCLUSION No significant improvement in the clinical pregnancy rates was observed when GnRH antagonists were used in $\mathrm{COH}+\mathrm{IUI}$ cycles, despite the significant increase in the number of follicles that were $>16 \mathrm{~mm}$ on HCG trigger day.

Keywords: GnRH antagonist, intrauterine insemination, ovarian stimulation, pregnancy rates

\section{INTRODUCTION}

Intrauterine insemination (IUI) is widely used as an infertility treatment modality. The success of assisted reproductive technologies is dependent on appropriate patient selection and adequate development of oocytes. Compared to clomiphene citrate ovarian stimulation, gonadotropin ovarian stimulation with IUI results in higher pregnancy rates. Moreover, the combination of controlled ovarian hyperstimulation $(\mathrm{COH})+\mathrm{IUI}$ has been shown to increase the fecundity of the cycle as compared to IUI alone. ${ }^{(1,2)}$ There are variations in the reported clinical pregnancy rates of $\mathrm{COH}+\mathrm{IUI}$ cycles; these may be attributed to differences in the aetiology and duration of infertility, sperm preparation technique, the number of sperms injected, the number of inseminations per cycle, cycle monitoring, IUI timing and the ovarian stimulation protocol selected. ${ }^{(2-5)}$

Although low-dose protocols with recombinant folliclestimulating hormone $(\mathrm{rFSH})$ are used during $\mathrm{COH}+\mathrm{IUI}$ cycles, ${ }^{(6)}$ multifollicular development may occur and result in a sudden increase in serum estradiol (E2) levels, which can result in a premature luteinising hormone $(\mathrm{LH})$ peak (before follicular maturation) and revocation of the IUI. ${ }^{(7)}$ Gonadotropin-releasing hormone $(\mathrm{GnRH})$ analogues lead to the desensitisation of pituitary GnRH receptors and, via this phenomenon, block endogenous $\mathrm{LH}$ increase. The use of GnRH analogues has been reported to lower premature LH peak to approximately $2 \%$ and subsequently increase pregnancy rates. ${ }^{(8)}$ For more than ten years, the use of gonadotropins with $\mathrm{GnRH}$ agonists has been the most frequently applied treatment protocol for reducing the incidence of premature LH peak. ${ }^{(9)}$ However, this treatment protocol, which needs 2-3 weeks of desensitisation time, also increases the amount of gonadotropins used, the risk of ovarian hyperstimulation syndrome and the duration of treatment. During the desensitisation period, patients are also exposed to side effects such as hot flushes, headaches, vaginal dryness and bleeding. These drawbacks have resulted in the recent removal of IUI treatment protocols, including GnRH agonists. A Cochrane review on ovarian stimulation protocols for IUI advised against the use of $\mathrm{GnRH}$ agonists in $\mathrm{COH}$ with low-dose exogenous FSH (i.e. mild $\mathrm{COH}$ ). ${ }^{(10)}$

$\mathrm{GnRH}$ antagonists, which are produced through the exchange of amino acids of $\mathrm{GnRH}$ with other molecules at multiple points, bind to GnRH receptors with high affinity. ${ }^{(11)}$ These antagonistic molecules prevent the release of endogenous gonadotropin and are currently being considered to replace GnRH agonists due to their positive pharmacokinetic and pharmacodynamic properties. ${ }^{(11)}$ The advantages of $\mathrm{GnRH}$ antagonists include absence of the first flare-up effect, reduced risk of oestrogen deficiency syndrome (since there is no need for long-term desensitisation), sufficient $\mathrm{LH}$ blockage in a short duration of time, dose-dependent effect

${ }_{1}^{1}$ Department of Obstetrics and Gynecology, Bezmialem Vakif University, School of Medicine, ${ }^{2}$ Suleymaniye Women Health Training Hospital, Istanbul, Turkey Correspondence: Professor Ramazan Dansuk, Chairman, Department of Obstetrics and Gynecology, Bezmialem Vakif University, Adnan Menderes Bulvari Vatan Caddesi 34093 Fatih, Istanbul, Turkey. rdansuk@hotmail.com 
and fast withdrawal from antagonist effects, which make $\mathrm{GnRH}$ antagonists superior to $\mathrm{GnRH}$ agonists. ${ }^{(6,12)}$ Furthermore, studies on in vitro fertilisation (IVF) and intracytoplasmic sperm injection protocols have shown that treatment duration and the total dose of gonadotropin used are reduced when antagonists are used in the protocol. ${ }^{(13)}$ It has also been shown that GnRH antagonists are associated with a low risk of high-order multiple pregnancies when standard strict criteria are applied for cycle cancellation. ${ }^{(14)}$

Although there is controversy regarding the effectiveness of $\mathrm{GnRH}$ antagonists in low-dose ovarian hyperstimulation protocols, if current and future studies show that these antagonists are able to improve pregnancy rates, the frequency of their use may increase in the future. In the present study, we studied the effect of $\mathrm{GnRH}$ antagonists in IUI cycles, in which rFSH had been used for $\mathrm{COH}$.

\section{METHODS}

The present study was conducted between 1 April 2009 and 10 June 2009 in Suleymaniye Maternity and Women's Disease Research and Teaching Hospital's outpatient clinics for infertility, the most visited infertility clinics in Istanbul, Turkey. The study was approved by the local ethics committee.

Patients who met the following criteria were eligible for inclusion in the study: (a) indication for treatment with IUI (e.g. unexplained infertility, mild male factor, minimal or mild [stage I or II] endometriosis); (b) a history of two cycles of ovarian stimulation treatment with clomiphene citrate; (c) determination of tubal patency by hysterosalpingography (HSG) or laparoscopy; (d) age 18-39 years; (e) body mass index (BMI) 18-39 kg/m²; (f) regular menstrual cycles (25-32 days); (g) basal $\mathrm{FSH}<10 \mathrm{IU} / \mathrm{mL}$, and normal levels of thyroid-stimulating hormone and prolactin; and (h) at least $5 \mathrm{million} / \mathrm{mL}$ sperm count and $5 \%$ normal morphology on Kruger test. Patients who had clinically significant systemic or endocrine disease, a diagnosis of polyp, submucous myoma, uterine septum or any other spaceoccupying lesion during HSG or office hysteroscopy evaluation and previous IUI were excluded from the study.

A total of 126 patients agreed to participate in the study and informed consent was obtained. However, only 108 patients were included in the data analysis; 18 patients did not have the appropriate response to treatment, were lost to follow-up or did not have timely sonography. If any one of the following criteria was met, the treatment cycle would be cancelled: (a) premature luteinisation; (b) progesterone level $>1.7 \mathrm{ng} / \mathrm{dL}$ during $\mathrm{COH}$ on the day of human chorionic gonadotropin (HCG) trigger for ovulation; (c) premature LH peak; (d) LH level > $12.1 \mathrm{mlU} / \mathrm{mL}$ on HCG trigger day; (e) probability of multiple gestations due to the presence of more than four follicles $>15 \mathrm{~mm}$; and (f) poor response to treatment (i.e. no follicle $>10 \mathrm{~mm}$ ).

The patients were randomly divided into two groups (group A and group B) using an online research randomiser software (www. randomizer.org). The patients in group $\mathrm{A}$ received $\mathrm{rFSH}$ and $\mathrm{GnRH}$ antagonist for $\mathrm{COH}$, while those in group $\mathrm{B}$ received only rFSH. In both groups, ovarian stimulation was started on the third day of the menstrual cycle. We used rFSH (GONAL-f ${ }^{\circledR}$; Merck
Serono, Rome, Italy) for ovarian stimulation. When calculating the starting dose for each patient, the expected ovarian response was taken into consideration. Most of the patients were prescribed $75 \mathrm{IU} / \mathrm{mL} \mathrm{rFSH}$ subcutaneous injections around the umbilicus in the mornings. New dosage was adjusted according to the ovarian response (examined through follicle number and dimension, and serum E2 levels on the sixth or seventh day of stimulation) and stimulation was continued until the day of HCG.

When the dominant follicle reached a diameter of $>14 \mathrm{~mm}$, once-daily subcutaneous injection of the $\mathrm{GnRH}$ antagonist, Cetrorelix (cetrotide flacon $0.25 \mathrm{mg}$ ), was added to the protocol for group A patients; the use of the $\mathrm{GnRH}$ antagonist requires continuation of gonadotropin. Cetrorelix was continued until the day of insemination. In both groups, when one, two or three follicles reached a diameter of $17 \mathrm{~mm}, 10,000$ IU HCG (Pregnyl ampoule 5,000 IU, 2 ampoules) was injected intramuscularly, with the aim of triggering ovulation. After a mean duration of 35.5 (range 34-38) hours, IUI was performed. Serum $\beta$ HCG was measured 14 days after ovulation, and the $\beta$ HCG test was repeated in patients who tested positive. To determine clinical pregnancy, transvaginal sonography was used to confirm viability at 5-7 weeks of gestation. In both groups, every couple was subjected to only one cycle of treatment. The following efficacy parameters were compared between groups A and B: (a) primary outcome measure - clinical pregnancy rate; and (b) secondary outcome measures - duration of induction, total dose of $\mathrm{rFSH}$ used, folliculometry results on HCG day (i.e. number of follicles $>15 \mathrm{~mm}$ ) and endometrial thickness on HCG day.

Statistical analyses were performed using Number Cruncher Statistical System 2007 and Power Analysis and Sample Size 2008 (NCSS, LCC; Kaysville, UT, USA). Student's t-test was used for comparing continuous variables that showed normal distribution, while Mann-Whitney $U$ test was used for variables that did not follow a normal distribution. To compare qualitative data, chisquare test and Fisher's exact test were used. Results were evaluated using 95\% confidence interval and $p<0.05$ significance level.

\section{RESULTS}

A total of 126 patients agreed to participate in the study - 61 patients were assigned to group $\mathrm{A}$ and 65 were assigned to group $\mathrm{B}$. In group A, 16 patients were excluded from the data analysis seven were either lost to follow-up or did not have timely sonography, three had insufficient response to treatment (no development of a dominant follicle), and six patients had cycle cancellation due to $>4$ follicles measuring $>15 \mathrm{~mm}$. In group $B$, one patient was lost to follow-up and one did not develop any follicles. Finally, 45 patients in group A and 63 patients in group B were included in the assessment.

The mean age of the 108 patients included in the final analysis was $31.84 \pm 3.73$ (range 21-37) years and mean BMI was $24.40 \pm 1.88$ (range 19-26) kg/m². The differences in mean age and BMI between groups A and B were not statistically significant. Table I shows the clinical characteristics, including the duration of infertility, of the patients in both groups. The mean duration of $\mathrm{rFSH}$ stimulation was $9.49 \pm 2.74$ days and $9.73 \pm 2.82$ days 
Table I. Clinical characteristics of the patients $(n=108)$.

\begin{tabular}{|c|c|c|c|}
\hline \multirow[t]{2}{*}{ Characteristic } & \multicolumn{2}{|c|}{ Mean \pm SD } & \multirow[t]{2}{*}{ p-value* } \\
\hline & Group A ( $n=45)$ & Group B (n = 63) & \\
\hline Duration of infertility (yr) & $8.39 \pm 4.44$ & $8.02 \pm 4.60$ & 0.681 \\
\hline No. of follicles $>16 \mathrm{~mm}$ on HCG trigger day ${ }^{+}$ & $1.86 \pm 0.59(2)$ & $1.58 \pm 0.67(1)$ & 0.016 \\
\hline Duration of rFSH stimulation (day) & $9.49 \pm 2.74$ & $9.73 \pm 2.82$ & 0.659 \\
\hline Total rFSH dose (IU) & $988.33 \pm 323.41$ & $871.83 \pm 343.44$ & 0.078 \\
\hline Endometrial thickness (mm) & $9.26 \pm 1.66$ & $9.45 \pm 1.53$ & 0.535 \\
\hline
\end{tabular}

* Statistical analysis for no. of follicles was performed using Mann-Whitney $U$ test, while statistical analysis for all other parameters was performed using Student's $t$-test. + Data presented as mean \pm SD (median). HCG: human chorionic gonadotropin; rFSH: recombinant follicle-stimulating hormone; SD: standard deviation

in group A and group B, respectively. There was no significant difference in the duration of $\mathrm{rFSH}$ stimulation between the two groups. In other words, the use of GnRH antagonist did not prolong the duration of $\mathrm{rFSH}$ stimulation. Although the mean total $\mathrm{rFSH}$ dose administered was slightly higher in group $\mathrm{A}$ than in group B (988.33 IU vs. $871.83 \mathrm{IU})$, the difference was not statistically significant. However, the median number of follicles $>16 \mathrm{~mm}$ on HCG trigger day was significantly higher in group A than in group B (1.86 vs. 1.58; $\mathrm{p}<0.05)$.

The mean endometrial thickness in group A was $9.26 \mathrm{~mm}$ and that in group B was 9.45 mm; however, this difference was not statistically significant. No statistically significant differences were observed in the number of cancelled cycles due to premature luteinisation (none in group A, two in group B) and the rate of clinical pregnancy $(8.9 \%$ in group $A, 7.9 \%$ in group $B)$.

\section{DISCUSSION}

There is no consensus regarding the use of $\mathrm{GnRH}$ antagonists during $\mathrm{COH}+\mathrm{IUI}$ cycles in the literature. Although earlier investigations have shown the benefits of these molecules, ${ }^{(6,15-18}$ subsequent studies have failed to confirm them. ${ }^{(19-22)}$ In 2005, Gómez-Palomares et al concluded from their prospective randomised study that the addition of $\mathrm{GnRH}$ antagonists to $\mathrm{COH}+$ IUI cycles significantly increased pregnancy rates. ${ }^{(15)}$ In 2008, another study by the same authors reported that the markedly better pregnancy rates seen in patients who were administered $\mathrm{GnRH}$ antagonists could be due to the multifollicular development of mature (> $18 \mathrm{~mm}$ ) follicles, as $\mathrm{GnRH}$ antagonists allow for the growth of intermediate follicles without the risk of premature luteinisation. ${ }^{(17)}$ Subsequent studies, however, have failed to show the effect of $\mathrm{GnRH}$ antagonists on multifollicular development in $\mathrm{COH}+\mathrm{IUI}$ cycles. ${ }^{(6,19)}$ In a study by Allegra et al, none of the women with an $\mathrm{LH}$ level $>10 \mathrm{mIU} / \mathrm{mL}$ achieved pregnancy, and the authors concluded that $\mathrm{GnRH}$ antagonists improved pregnancy rates by preventing the occurrence of premature luteinisation. ${ }^{(6)}$

A study by Lambalk et al showed that although $\mathrm{GnRH}$ antagonists may reduce the incidence of premature luteinisation, these antagonists do not positively affect pregnancy rate. ${ }^{(16)}$ Crosignani et al's study also failed to prove that the use of $\mathrm{GnRH}$ antagonists is beneficial in IUI cycles; the authors speculated that the benefit of $\mathrm{GnRH}$ antagonists in preventing premature $\mathrm{LH}$ surge was countered by the unfavourable effects of $\mathrm{GnRH}$ antagonists, which were not well understood then. ${ }^{(19)}$ In another study, Martinez-Salazar et al concluded that, while the universal use of GnRH antagonists in $\mathrm{COH}+\mathrm{IUI}$ cycles does not increase pregnancy rates, it may benefit a specific subset of patients who have premature luteinisation or high progesterone concentrations in a previous failed IUI. ${ }^{(20)}$ It should be noted that Martinez-Salazar et al did not evaluate the $\mathrm{LH}$ and progesterone levels during the cycle when GnRH antagonists were used and, therefore, could not determine the exact effect GnRH antagonists had on premature luteinisation. ${ }^{(20)}$ In a multicentre, double-blinded, randomised controlled trial that involved four academic and eight institutional hospitals, Cantineau et al concluded that the addition of $\mathrm{GnRH}$ antagonists to standard IUI treatment causes the treatment to be unnecessarily complex, and that GnRH antagonists should thus not be applied in daily practice. ${ }^{(21)}$ In fact, a recent trial evaluating the effectiveness of GnRH antagonists in IUI cycles was discontinued because of the lower pregnancy rates observed in the $\mathrm{GnRH}$ antagonist group. ${ }^{(22)}$ Similarly, the 2011 Cochrane review on ovarian stimulation protocols for IUI cycles did not reach a definite conclusion on the use of GnRH antagonists; while the use of GnRH antagonists was not advised in mild $\mathrm{COH}$ cycles, it was recommended that the utility of $\mathrm{GnRH}$ be determined in future trials. ${ }^{(10)}$

In the present study, we were not able to prove the benefits of $\mathrm{GnRH}$ antagonists in clinical pregnancy rates. However, $\mathrm{GnRH}$ antagonists may still be useful in specific infertile subpopulations, such as women with polycystic ovarian syndrome or women in whom $\mathrm{COH}+\mathrm{IUI}$ cycle has to be converted to an IVF cycle. ${ }^{(23,24)}$ The use of $\mathrm{GnRH}$ antagonists has also been shown to be effective in manipulating follicular development, so that the insemination process can be suspended on weekends without an apparent decrease in pregnancy rates. ${ }^{(25,26)}$

The present study was not without limitations. The number of women enrolled in the study small; nevertheless, other studies investigating the same subject have also enrolled small numbers of patients. ${ }^{(6,16,19)}$ Another limitation was the difference between the number of patients in the two groups due to asymmetric dropouts. This, however, did not affect the significance of the results of statistical analyses performed.

In conclusion, the present study revealed no significant improvement in clinical pregnancy rates when GnRH antagonists were used during $\mathrm{COH}+\mathrm{IUI}$ cycles, despite a significant increase in the number of follicles $>16 \mathrm{~mm}$ on the HCG trigger day. Larger randomised controlled trials are required to determine whether liberal use of GnRH antagonists during IUI cycles should be recommended. 


\section{REFERENCES}

1. Hughes EG. The effectiveness of ovulation induction and intrauterine insemination in the treatment of persistent infertility: a meta-analysis. Hum Reprod 1997; 12:1865-72.

2. Guzick DS, Carson SA, Coutifaris C, et al. Efficacy of superovulation and intrauterine insemination in the treatment of infertility. National Cooperative Reproductive Medicine Network. N Engl J Med 1999; 340:177-83.

3. Bagis T, Haydardedeoglu B, Kilicdag EB, et al. Single versus double intrauterine insemination in multi-follicular ovarian hyperstimulation cycles: a randomized trial. Hum Reprod 2010; 25:1684-90.

4. Cantineau AE, Heineman MJ, Cohlen BJ. Single versus double intrauterine insemination (IUI) in stimulated cycles for subfertile couples. Cochrane Database Syst Rev 2003; CD003854.

5. Hughes EG. Stimulated intrauterine insemination is not a natural choice for the treatment of unexplained subfertility. 'Effective treatment' or 'not a natural choice'? Hum Reprod 2003; 18:912-14.

6. Allegra A, Marino A, Coffaro F, et al. GnRH antagonist-induced inhibition of the premature LH surge increases pregnancy rates in IUI-stimulated cycles. A prospective randomized trial. Hum Reprod 2007; 22:101-8.

7. Ragni G, Somigliana E, Vegetti W. Timing of intrauterine insemination: where are we? Fertil Steril 2004; 82:25-6.

8. Edelstein MC, Seltman HJ, Cox BJ, et al. Progesterone levels on the day of human chorionic gonadotropin administration in cycles with gonadotropinreleasing hormone agonist suppression are not predictive of pregnancy outcome. Fertil Steril 1990; 54:853-7.

9. Manzi DL, Dumez S, Scott LB, Nulsen JC. Selective use of leuprolide acetate in women undergoing superovulation with intrauterine insemination results in significant improvement in pregnancy outcome. Fertil Steril 1995; 63:866-73.

10. Cantineau AE, Cohlen BJ, Heineman MJ. Ovarian stimulation protocols (anti-oestrogens, gonadotrophins with and without $\mathrm{GnRH}$ agonists/ antagonists) for intrauterine insemination (IUI) in women with subfertility. Cochrane Database Syst Rev 2007; CD005356.

11. Olivennes F, Cunha-Filho JS, Fanchin R, Bouchard P, Frydman R. The use of GnRH antagonists in ovarian stimulation. Hum Reprod Update 2002; 8:279-90.

12. Diedrich K, Diedrich C, Santos E, et al. Suppression of the endogenous luteinizing hormone surge by the gonadotrophin-releasing hormone antagonist Cetrorelix during ovarian stimulation. Hum Reprod 1994; 9:788-91.

13. Griesinger G, Felberbaum R, Diedrich K. GnRH antagonists in ovarian stimulation: a treatment regimen of clinicians' second choice? Data from the German national IVF registry. Hum Reprod 2005; 20:2373-5.

14. Ragni G, Caliari I, Nicolosi AE, et al. Preventing high-order multiple pregnancies during controlled ovarian hyperstimulation and intrauterine insemination: 3 years' experience using low-dose recombinant follicle-stimulating hormone and gonadotropin-releasing hormone antagonists. Fertil Steril 2006; 85:619-24.

15. Gómez-Palomares JL, Juliá B, Acevedo-Martín B, et al. Timing ovulation for intrauterine insemination with a GnRH antagonist. Hum Reprod 2005; 20:368-72.

16. Lambalk CB, Leader A, Olivennes F, et al. Treatment with the GnRH antagonist ganirelix prevents premature LH rises and luteinization in stimulated intrauterine insemination: results of a double-blind, placebocontrolled, multicentre trial. Hum Reprod 2006; 21:632-9.

17. Gómez-Palomares JL, Acevedo-Martín B, Chávez M, et al. Multifollicular recruitment in combination with gonadotropin-releasing hormone antagonist increased pregnancy rates in intrauterine insemination cycles. Fertil Steril 2008; 89:620-4.

18. Kosmas IP, Tatsioni A, Kolibianakis EM, et al. Effects and clinical significance of $\mathrm{GnRH}$ antagonist administration for IUI timing in FSH superovulated cycles: a meta-analysis. Fertil Steril 2008; 90:367-72.

19. Crosignani PG, Somigliana E; Intrauterine Insemination Study Group. Effect of $\mathrm{GnRH}$ antagonists in FSH mildly stimulated intrauterine insemination cycles: a multicentre randomized trial. Hum Reprod 2007; 22:500-5.

20. Martinez-Salazar J, Cerrillo M, Quea G, Pacheco A, Garcia-Velasco JA. $\mathrm{GnRH}$ antagonist ganirelix prevents premature luteinization in IUI cycles: rationale for its use. Reprod Biomed Online 2009; 19:156-61.

21. Cantineau AE, Cohlen BJ, Klip H, Heineman MJ; Dutch IUI Study Group Collaborators. The addition of $\mathrm{GnRH}$ antagonists in intrauterine insemination cycles with mild ovarian hyperstimulation does not increase live birth rates--a randomized, double-blinded, placebo-controlled trial. Hum Reprod 2011; 26:1104-11.

22. Kamath MS, R R, Bhave P, et al. Effectiveness of GnRH antagonist in intrauterine insemination cycles. Eur J Obstet Gynecol Reprod Biol 2013; 166:168-72

23. Ertunc D, Tok EC, Savas A, Ozturk I, Dilek S. Gonadotropin-releasing hormone antagonist use in controlled ovarian stimulation and intrauterine insemination cycles in women with polycystic ovary syndrome. Fertil Steril 2010; 93:1179-84

24. Quaas AM, Missmer SA, Ginsburg ES. Gonadotropin-releasing hormone antagonist use is associated with increased pregnancy rates in ovulation induction-intrauterine insemination to in vitro fertilization conversions, independent of age and estradiol level on the day of human chorionic gonadotropin administration. Fertil Steril 2010; 93:605-8.

25. Checa MA, Prat M, Robles A, Carreras R. Use of gonadotropin-releasing hormone antagonists to overcome the drawbacks of intrauterine insemination on weekends. Fertil Steril 2006; 85:573-7.

26. Matorras R, Ramón O, Expósito A, et al. Gn-RH antagonists in intrauterine insemination: the weekend-free protocol. J Assist Reprod Genet 2006; 23:51-4. 